\title{
3 SINTOMAS FÍSICOS E PSICOLÓGICOS DO ESTRESSE EM PESSOAS VIVENDO COM O VÍRUS DA IMUNODEFICIÊNCIA HUMANA
}

\author{
Elizabete Melo ${ }^{1}$; Marcela Antonini ${ }^{2}$ Christefany Costa ${ }^{3}$; Priscila Pontes ${ }^{4}$; Lucilene Cardoso ${ }^{5}$; Elucir Gir ${ }^{6}$; Renata Reis ${ }^{7}$
}

\section{RESUMO}

INTRODUÇÃO: O viver com o HIV, além de ser uma doença sem cura e com uma alta carga estigmatizadora, é considerada um fenômeno social que convive com o sofrimento físico representando uma alta fonte de estresse.

OBJETIVO: Avaliar a prevalência, fatores associados e sintomas físicos e psicológicos do estresse em pessoas vivendo com HIV, comparando-o segundo o sexo.

MÉTODOS: Trata-se de um estudo analítico de corte transversal, realizado com pessoas que vivem com HIV em atendimento ambulatorial na região sudeste do Brasil. Foi utilizado questionário para caracterização sociodemográfica e clínica, e para avaliação dos sintomas de estresse foi utilizado o Inventário de Sintomas de Stress de Lipp (ISSL), o qual identifica a presença de estresse, a sintomatologia predominante e a fase em que o paciente se encontra. Para análise foi aplicado o teste de associação Qui-quadrado, adotando p<0,05.

RESULTADOS: Participaram do estudo 340 pessoas, das quais 47,6\% foram diagnosticadas com estresse. Houve associação do estresse com a idade $(\mathrm{p}=0,018)$, tempo de diagnóstico $(\mathrm{p}=0,025)$ e tempo de tratamento $(\mathrm{p}=0,016)$. As mulheres apresentaram maior frequência de sintomas de estresse nas fases de alerta $(60,8 \%)$, quase-exaustão $(75,0 \%)$ e de exaustão $(54,8 \%)$, com predomínio dos sintomas físicos, destacando a impossibilidade de trabalhar $(\mathrm{p}=0,03)$, a vontade de fugir de tudo $(\mathrm{p}=0,004)$ e pensar/falar sobre o mesmo assunto $(\mathrm{p}=0,02)$.

CONCLUSÕES: O presente estudo evidenciou uma alta prevalência do estresse em PVHIV, com associação entre a idade, o tempo de diagnóstico e o tempo de tratamento com antirretroviral; e predomínio entre sinais e sintomas nas mulheres.

\section{PALAVRAS-CHAVE: Estresse psicológico; Saúde mental; Infecções por HIV}

\section{RESUMEN}

\section{"Síntomas físicos y psicológicos del estrés en las personas que viven con el virus de la inmunodeficiencia humana"}

INTRODUCCIÓN: Vivir con VIH, además de ser una enfermedad sin cura y con una alta carga de estigmatización, se considera un fenómeno social que coexiste con el sufrimiento físico que representa una gran fuente de estrés. OBJETIVO: Evaluar la prevalencia, los factores asociados y los síntomas físicos y psicológicos del estrés en personas con VIH comparándolo por género. METODOLOGÍA: Este es un estudio analítico transversal realizado con personas que viven con VIH en atención ambulatoria en el sudeste de Brasil. Se utilizó un cuestionario para la caracterización sociodemográfica y clínica, y para la evaluación de los síntomas de estrés, se utilizó el Inventario de Síntomas de Estrés de Lipp (ISSL), que identifica la presencia de estrés, la sintomatología predominante y la fase del paciente. La prueba de asociación de chi-cuadrado se aplicó para el análisis, adoptando $\mathrm{p}<0,05$.

RESULTADOS: 340 personas participaron en el estudio, de los cuales el 47,6\% fueron diagnosticados con estrés. El estrés se asoció con la edad $(\mathrm{p}=0,018)$, el tiempo desde el diagnóstico $(p=0,025)$ y el tiempo de tratamiento $(p=0,016)$. Las mujeres tuvieron una mayor frecuencia de síntomas de estrés en las fases de alerta $(60,8 \%)$, casi agotamiento $(75,0 \%)$ y agotamiento $(54,8 \%)$, con predominio de síntomas físicos, destacando la imposibilidad de trabajar $(\mathrm{p}=0,03)$, el deseo de alejarse de todo $(\mathrm{p}=0,004)$ y pensar / hablar sobre el mismo tema $(\mathrm{p}=0,02)$.

CONCLUSIONES: El presente estudio mostró una alta prevalencia de estrés, con una asociación entre la edad, el tiempo desde el diagnóstico y la duración del tratamiento antirretroviral; y predominio entre signos y síntomas en mujeres.

DESCRIPTORES: Estrés psicológico; Salud mental; Infecciones por VIH

\section{ABSTRACT \\ "Physical and psychological symptoms of stress in people living with the human immunodeficiency virus"}

BACKGROUND: Living with HIV, besides being a disease without cure and with a high stigmatizing burden, is considered a social phenomenon that coexists with physical suffering representing a high source of stress.

AIM: To evaluate the prevalence, associated factors, and physical and psychological symptoms of stress in people living with HIV by comparing stress by sex.

METHODS: This is a cross-sectional analytical study conducted with people living with HIV in outpatient care in southeastern Brazil. A questionnaire was used for sociodemographic and clinical characterization; and for the evaluation of stress symptoms, the Lipp Stress Symptom Inventory (ISSL) was used, which identifies the presence of stress, the predominant symptomatology and the phase of the patient. The chi-square association test was applied for analysis, adopting $\mathrm{p}<0.05$.

RESULTS: 340 people participated in the study, of which $47.6 \%$ were diagnosed with stress. Stress was associated with age $(\mathrm{p}=0.018)$, time of diagnosis $(\mathrm{p}=0.025)$ and time of treatment $(\mathrm{p}=0.016)$. Women presented a higher frequency of stress symptoms in the alert (60.8\%), near-exhaustion (75.0\%) and exhaustion $(54.8 \%)$ phases, with a predominance of physical symptoms, highlighting the impossibility of working $(\mathrm{p}=0.03)$, the desire to escape from everything $(\mathrm{p}=0.004)$ and think/talk about the same subject $(\mathrm{p}=0.02)$.

CONCLUSIONS: The present study showed a high prevalence of stress, with an association between age, time since diagnosis and duration of antiretroviral treatment; and predominance between signs and symptoms of stress in women.

\section{KEYWORDS: Stress, psychological; Mental health; HIV Infec- tions}

Submetido em 31-03-2019

Aceite em 01-10-2019

\footnotetext{
1 Enfermeira; Estudante de Doutorado na Universidade de São Paulo, Escola de Enfermagem de Ribeirão Preto, Ribeirão Preto (SP), Brasil, elizabetemelo@usp.br 2 Estudante de Graduação em Enfermagem na Universidade de São Paulo, Escola de Enfermagem de Ribeirão Preto, Ribeirão Preto (SP), Brasil, marcela.antonini@usp.br 3 Enfermeira; Estudante de Doutorado na Universidade de São Paulo, Escola de Enfermagem de Ribeirão Preto, Ribeirão Preto (SP), Brasil, christefany.costa@usp.br 4 Enfermeira; Estudante de Mestrado na Universidade de São Paulo, Escola de Enfermagem de Ribeirão Preto, Ribeirão Preto (SP), Brasil, priscilapontes@usp.br 5 Enfermeira; Professora Associada na Universidade de São Paulo, Escola de Enfermagem de Ribeirão Preto, Ribeirão Preto (SP), Brasil, lucilene@eerp.usp.br 6 Enfermeira; Professora Titular naa Universidade de São Paulo, Escola de Enfermagem de Ribeirão Preto, Ribeirão Preto (SP), Brasil, egir@eerp.usp.br

7 Enfermeira; Professora Associada na Universidade de São Paulo, Escola de Enfermagem de Ribeirão Preto, Ribeirão Preto (SP), Brasil, rkreis@eerp.usp.br
}

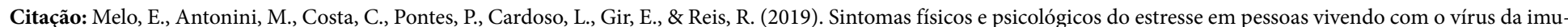
nodeficiência humana. Revista Portuguesa de Enfermagem de Saúde Mental (22), 19-26. 


\section{INTRODUÇÃO}

No Brasil, a disponibilidade da terapia antirretroviral (TARV) pelo Sistema Único de Saúde (SUS), para as pessoas vivendo com o vírus da imunodeficiência humana (PVHIV), desde 1996, resultou em uma redução nos números de morbimortalidade por doenças oportunistas associadas ao HIV, e consequentemente diminuiu o número de internações hospitalares e aumentou a expectativa de vida, promovendo uma melhoria na qualidade de vida das pessoas acometidas (Foresto et al., 2017).

Todas estas implicações refletem na importância da avaliação da efetividade em aderir ao tratamento, principalmente por influenciar diretamente nas condições clínicas e psicológicas das PVHIV (Galvão, Soares, Pedrosa, Fiuza \& Lemos, 2015).

Segundo o The Jointed United Nations Programme on HIV/AIDS (UNAIDS), até 2017 existiam cerca de 37 milhões de PVHIV em todo o mundo, e destes, 21,7 milhões encontravam-se em terapia antirretroviral. No Brasil, até junho de 2018 foram notificados 982.129 casos de aids, dos quais $65,5 \%$ são homens e cerca de $72 \%$ encontram-se em TARV (Brasil, 2018; The Jointed United Nations Programme on HIV/AIDS [UNAIDS], 2016). $\mathrm{O}$ viver com HIV, além de ser uma condição crônica e com uma alta carga estigmatizadora, é considerado um fenômeno social que convive com o sofrimento físico representando uma alta fonte de tensão. Além disso, as PVHIV vivenciam experiências de intolerância, medo, preconceito, exclusão social, e consequentemente, estas situações trazem inúmeras incertezas para o seu futuro (Verhey, Gibson, Brakarsh, Chibanda \& Seedat, 2018). Neste contexto, destaca-se ainda que os impactos psicossociais levam à reflexão e reavaliação das crenças, vínculos profissionais e afetivos, gerando repercussões na adesão ao tratamento, podendo despertar diversos sentimentos associados à ansiedade, depressão, raiva, estresse entre outros que influenciam na evolução da doença debilitando cada vez mais o sistema imunológico e aumentando a vulnerabilidade de desenvolver outras comorbidades (Calvetti, Giovelli, Gauer \& Moraes, 2016; Cardoso, 2013).

Entende-se que a infecção pelo HIV apresenta características de uma condição crônica com curso incerto, uma vez que implica num alto nível de estresse ligado à readequação social e psicológica, pois as PVHIV enfrentam as diversas fases do tratamento permeado por estigmas, dúvidas e medos que levam a um constante estresse relacionado com a descoberta do diagnóstico, a mudança dos hábitos de vida, o uso de terapia medicamentosa contínua e a revelação do diagnóstico para a família, amigos e/ou parceiro (Reis et al., 2017).

Estudos já identificaram os mecanismos moleculares específicos que modulam o efeito induzido pelo estresse na dinâmica neuroendócrina da patogenia do HIV, evidenciando a existência de uma associação entre os fatores psicossociais e a progressão da infecção pelo HIV (Collado-Hidalgo, Sung \& Cole, 2006; Sloan et al., 2007), e neste contexto, alerta-se para uma maior ocorrência de transtornos psíquicos em PVHIV do que na população geral (Brasil, 2017; Pokhrel et al., 2017; Ursoiu, Moleriu, Lungeanu \& Puschită, 2018).

O estresse é considerado um dos males da atualidade, e pode ser definido por um conjunto de respostas fisiológicas específicas, com a finalidade de adaptação (Selye, 1956), afetando $90 \%$ da população mundial (StultsKolehmainen, Bartholomew \& Sinha, 2014).

Ele surge a partir de agentes estressores que se referem a qualquer situação geradora de um estado emocional forte que leva a uma quebra da homeostase interna, exigindo alguma adaptação (Lipp e Malagris, 1989).

A partir disso, a literatura aponta que os estressores diários são capazes de corroer a resiliência, reduzindo os mecanismos eficazes de enfrentamento, gerando um impacto na saúde mental (Verhey et al., 2018). E a partir disso é necessário conhecer o nível de estresse das PVHIV, a fim de promover intervenções resolutivas por meio de um atendimento integral.

Nota-se que são escassos os estudos na literatura que avaliaram como o estresse interfere na vida de PVHIV no Brasil, e, portanto, buscou-se com esse estudo avaliar a prevalência, fatores associados e os sintomas físicos e psicológicos do estresse em pessoas vivendo com HIV, comparando-o segundo o sexo.

\section{MÉTODOS}

Procedeu-se um estudo analítico de corte transversal. A amostra foi não probabilística consecutiva, constituída por 340 pessoas que vivem com HIV, selecionados por conveniência em cinco serviços de atendimento especializado para o atendimento a PVHIV, em nível ambulatorial na região sudeste do Brasil, no período de outubro de 2014 a outubro de 2016. Foram incluídos pacientes que conheciam sua condição sorológica, idade igual ou superior a 18 anos de ambos os sexos; em uso da terapia antirretroviral (TARV) (mínimo seis meses), acompanhado ambulatorialmente; e excluídos indivíduos em situações de confinamento e gestantes. 
Utilizou-se instrumento semiestruturado para caracterização sociodemográfica, clínica e hábitos de vida, construído para o estudo. Os dados sociodemográficos - sexo (masculino/feminino), idade (em anos completos), escolaridade (anos de estudo), estado civil (casado, solteiro, viúvo, separado), situação de trabalho (ativo/ inativo), orientação sexual (homossexual, heterossexual, bissexual), vida sexual ativa ( $\operatorname{sim} /$ não); e hábitos de vida - tabagismo ( $\operatorname{sim} /$ não, há quanto tempo, quantos cigarros/dia), etilismo ( $\operatorname{sim} /$ não, há quanto tempo, quantas vezes/semana), realiza atividade física (sim/ não, qual atividade, quantos dias na semana, quantas hrs/dia, há quanto tempo), atividades de lazer (sim/ não, qual atividade, quantos dias na semana, quantas hrs/dia, há quanto tempo) foram questionados ao paciente.

No prontuário foram coletados os dados referentes ao diagnóstico do HIV (tempo de diagnóstico em anos) e uso da TARV (tempo de uso de TARV, antirretroviral em uso) e as datas e os valores mais atuais dos exames de linfócito T CD4+ e carga viral.

Para avaliação dos sintomas de estresse foi utilizado o Inventário de Sintomas de Stress de Lipp (ISSL). O ISSL, instrumento validado no Brasil, tem sido utilizado em pesquisas e trabalhos clínicos na área do estresse. É um instrumento que tem como finalidade identificar o estresse, a sintomatologia predominante (física ou psicológica) e a fase em que ele se encontra, sendo definidas em quatro fases de manifestação: alerta, resistência, quase-exaustão e exaustão (Lipp e Guevara, 1994).

$\mathrm{O}$ instrumento é dividido em três quadros que representam os sinais e sintomas das últimas 24 horas, da última semana e do último mês, respectivamente. $\mathrm{E}$ cada um deles representa as fases de estresse, contendo sintomas físicos e psicológicos, conforme apresentado na Tabela 1 abaixo.

Tabela 1 - Características dos quadros contidos no Inventário de Sintomas de Stress de Lipp (ISSL), Ribeirão Preto, SP, 2018

\begin{tabular}{|l|c|c|c|}
\hline Sintomas & $\begin{array}{c}\text { Quadro 01 } \\
\text { Últimas 24 } \\
\text { horas }\end{array}$ & $\begin{array}{c}\text { Quadro 02 } \\
\text { Última semana }\end{array}$ & $\begin{array}{c}\text { Quadro 03 } \\
\text { Último mês }\end{array}$ \\
\hline Fases & Alerta & $\begin{array}{c}\text { Resistência e } \\
\text { Quase-exaustão }\end{array}$ & Exaustão \\
\hline $\begin{array}{l}\text { No Sintomas } \\
\text { físicos }\end{array}$ & 12 & 10 & 12 \\
\hline $\begin{array}{l}\text { No Sintomas } \\
\text { psicológicos }\end{array}$ & 03 & 05 & 11 \\
\hline
\end{tabular}

Fonte: LIPP, 2000
Para identificação do estresse e em qual fase o sujeito encontra-se, é realizado um somatório dos Sintomas Físicos (SF) e Psicológicos (SP) em cada quadro. No quadro 01 (alerta) é considerado alerta se o paciente apresentar mais de seis sintomas, no quadro 02 (resistência e quase-exaustão) se o paciente apresentar mais que três sintomas, o paciente está na fase de resistência, mas se apresentar mais que nove, é considerado quase-exaustão. No quadro 03, se o paciente apresentar mais de oito sintomas, ele está na fase de exaustão.

A análise dos dados foi realizada pelo software IBM SPSS (Statistical Package for Social Science) versão 22.0 para Windows. Realizou-se o teste Qui-Quadrado de Pearson para testar a associação entre as variáveis sociodemográficas e clínicas com a presença do estresse e entre os sintomas de estresse com o sexo. O nível de significância estabelecido foi de $5 \%(\mathrm{p}<0,05)$.

$\mathrm{O}$ presente estudo fundamentou-se na resolução brasileira do Conselho Nacional de Saúde (CNS) $466 / 2012$, que versa sobre os aspectos éticos das pesquisas que envolvem seres humanos. A coleta de dados foi realizada após a concordância dos indivíduos, mediante assinatura do Termo de Consentimento Livre e Esclarecido, em uma sala que resguardasse a privacidades dos mesmos. Na redação do referido termo constava a garantia do sigiloso das informações relacionadas à identidade pessoal, além da garantia de retirar-se do estudo a qualquer momento que julgar-se necessário. $\mathrm{O}$ projeto foi aprovado pelo Comitê de Ética da Escola de Enfermagem de Ribeirão Preto - Universidade de São Paulo, conforme protocolo no 749.563/2014.

\section{RESULTADOS}

Participaram do estudo 340 pessoas, sendo 58\% do sexo masculino, com média de idade de 44 anos, variando entre 20 e 75 anos. Com relação à orientação sexual, $70,3 \%$ declararam-se heterossexuais e $63,2 \%$ referiram ter vida sexual ativa no momento da entrevista.

O tempo médio de diagnóstico para o HIV foi de 10 anos $( \pm 7,16)$ e uma média de 7,6 anos de uso de TARV $( \pm 6,28)$. Além disso, $64,1 \%$ declarou sedentarismo e apenas 33,5\% realiza atividades de lazer (Tabela 2).

Após a análise, cerca de 47,6\% PVHIV tinham estresse e foi identificado que $5,8 \%$ dos entrevistados encontram-se na fase de alerta, com predomínio de sintomas físicos. Na fase de resistência foi encontrada uma prevalência de $47,7 \%, 12,8 \%$ de quase-exaustão e $23,3 \%$ na fase de exaustão, todas com predomínios de sintomas psicológicos. 


\begin{tabular}{|c|c|c|c|}
\hline Variáveis & Categoria & $\mathbf{n}$ & $\%$ \\
\hline \multirow{2}{*}{ Sexo } & Masculino & 197 & 57,9 \\
\hline & Feminino & 143 & 42,1 \\
\hline \multirow{5}{*}{ Idade (em anos) } & $<30$ & 52 & 15,3 \\
\hline & $30-39$ & 60 & 17,6 \\
\hline & $40-49$ & 116 & 34,1 \\
\hline & $50-59$ & 81 & 23,8 \\
\hline & $\geq 60$ & 31 & 9,1 \\
\hline \multirow{2}{*}{$\begin{array}{l}\text { Escolaridade } \\
\text { (anos de estudo) }\end{array}$} & $\leq 8$ & 167 & 49,1 \\
\hline & $>8$ & 173 & 50,9 \\
\hline \multirow{4}{*}{ Estado civil } & Casado & 120 & 35,3 \\
\hline & Solteiro & 147 & 43,2 \\
\hline & Viúvo & 34 & 10,0 \\
\hline & Separado & 39 & 11,5 \\
\hline \multirow{2}{*}{ Situação de trabalho } & Ativo & 193 & 56,8 \\
\hline & Inativo & 147 & 43,2 \\
\hline \multirow{3}{*}{ Orientação sexual } & Heterossexual & 239 & 70,3 \\
\hline & Homossexual & 82 & 24,1 \\
\hline & Bissexual & 19 & 5,6 \\
\hline \multirow{2}{*}{ Vida sexual ativa } & Sim & 215 & 63,2 \\
\hline & Não & 125 & 36,8 \\
\hline \multirow{2}{*}{ Tabagismo } & Sim & 109 & 32,1 \\
\hline & Não & 231 & 67,9 \\
\hline \multirow{2}{*}{ Etilismo } & Sim & 136 & 40,0 \\
\hline & Não & 204 & 60,0 \\
\hline \multirow{2}{*}{ Atividade física } & Inativo & 218 & 64,1 \\
\hline & Ativo & 122 & 35,88 \\
\hline \multirow{2}{*}{ Atividades de lazer } & Sim & 114 & 33,5 \\
\hline & Não & 226 & 66,5 \\
\hline \multirow{4}{*}{$\begin{array}{l}\text { Tempo de diagnósti- } \\
\text { co do HIV (em anos) }\end{array}$} & $<5$ & 121 & 35,6 \\
\hline & $5-10$ & 62 & 18,2 \\
\hline & $11-20$ & 132 & 38,8 \\
\hline & $>20$ & 25 & 7,4 \\
\hline \multirow{4}{*}{$\begin{array}{l}\text { Tempo de TARV } \\
\text { (anos) }\end{array}$} & $<5$ & 153 & 45,0 \\
\hline & $5-10$ & 68 & 20,0 \\
\hline & $11-20$ & 115 & 33,8 \\
\hline & $>20$ & 4 & 1,2 \\
\hline \multirow{2}{*}{$\begin{array}{l}\text { Células T CD4+ } \\
\text { (cel/mm3) }\end{array}$} & $\geq 200$ & 318 & 93,5 \\
\hline & $<200$ & 22 & 6,5 \\
\hline \multirow{2}{*}{$\begin{array}{l}\text { Carga viral } \\
\text { (cópias } / \mathrm{ml} \text { ) }\end{array}$} & $\leq 40$ & 275 & 80,9 \\
\hline & $>40$ & 65 & 19,1 \\
\hline \multirow{4}{*}{ Fases do estresse } & Alerta & 05 & 5,8 \\
\hline & Resistência & 41 & 47,7 \\
\hline & Quase-exaustão & 11 & 12,8 \\
\hline & Exaustão & 20 & 23,3 \\
\hline \multicolumn{2}{|l|}{ Total } & 340 & 100 \\
\hline
\end{tabular}

Tabela 3 - Associação entre a presença do estresse e as variáveis sociodemográficas e clínicas em PVHIV, n=340,

Ribeirão Preto, SP, Brasil, 2016

\begin{tabular}{|c|c|c|c|c|}
\hline \multirow{3}{*}{ Variáveis } & \multicolumn{3}{|c|}{ Estresse } & \multirow{3}{*}{$\mathrm{p}$} \\
\hline & \multirow{2}{*}{\begin{tabular}{|l} 
Não \\
178 \\
$(52,4 \%)$ \\
\end{tabular}} & \multirow{2}{*}{$\begin{array}{l}\text { Sim } \\
162 \\
(47,6 \%) \\
\end{array}$} & \multirow{2}{*}{$\frac{\text { Total }}{340(100 \%)}$} & \\
\hline & & & & \\
\hline \multicolumn{4}{|l|}{ Sexo } & \multirow{3}{*}{0,084} \\
\hline Masculino & $111(56,3)$ & $86(43,7)$ & $197(100)$ & \\
\hline Feminino & $67(46,9)$ & $76(53,1)$ & $143(100)$ & \\
\hline \multicolumn{4}{|l|}{ Idade (anos) } & \multirow{6}{*}{0,018} \\
\hline$<30$ & $22(46,8)$ & $25(53,2)$ & $47(100)$ & \\
\hline $30-39$ & $24(36,9)$ & $41(63,1)$ & $65(100)$ & \\
\hline $40-49$ & $62(53,4)$ & $54(46,6)$ & $116(100)$ & \\
\hline $50-59$ & $49(60,5)$ & $32(39,5)$ & $81(100)$ & \\
\hline$\geq 60$ & $21(67,7)$ & $10(32,3)$ & $31(100)$ & \\
\hline \multicolumn{4}{|c|}{ Escolaridade (anos de estudo) } & \multirow{3}{*}{0,926} \\
\hline$\leq 8$ & $87(52,1)$ & $80(47,9)$ & $167(100,0)$ & \\
\hline$>8$ & $91(52,6)$ & $82(47,4)$ & $173(100,0)$ & \\
\hline \multicolumn{4}{|l|}{ Estado Civil } & \multirow{5}{*}{0,254} \\
\hline Casado & $69(57,5)$ & $51(42,5)$ & $120(100,0)$ & \\
\hline Solteiro & $68(46,3)$ & $79(53,7)$ & $147(100,0)$ & \\
\hline Viúvo & $20(58,8)$ & $14(41,2)$ & $34(100,0)$ & \\
\hline Separado & $21(53,8)$ & $18(46,2)$ & $39(100,0)$ & \\
\hline \multicolumn{4}{|c|}{ Situação de trabalho } & \multirow{3}{*}{0,386} \\
\hline Ativo & $105(54,4)$ & $88(45,6)$ & $193(100,0)$ & \\
\hline Inativo & $73(49,7)$ & $74(50,3)$ & $147(100,0)$ & \\
\hline \multicolumn{4}{|c|}{ Orientação Sexual } & \multirow{4}{*}{0,087} \\
\hline Heterossexual & $134(56,1)$ & $105(43,9)$ & $239(100,0)$ & \\
\hline Homossexual & $37(45,1)$ & $45(54,9)$ & $82(100)$ & \\
\hline Bissexual & $7(36,8)$ & $12(63,2)$ & $19(100)$ & \\
\hline \multicolumn{4}{|c|}{ Vida Sexual ativa } & \multirow{3}{*}{0,317} \\
\hline Sim & $117(54,4)$ & $98(45,6)$ & $215(100)$ & \\
\hline Não & $61(48,8)$ & $64(51,2)$ & $125(100)$ & \\
\hline \multicolumn{4}{|c|}{ Tempo de diagnóstico (anos) } & \multirow{5}{*}{0,025} \\
\hline$<5$ & $50(46,3)$ & $58(53,7)$ & $108(100)$ & \\
\hline $5-10$ & $32(42,7)$ & $43(57,3)$ & $75(100)$ & \\
\hline $11-20$ & $80(60,6)$ & $52(39,4)$ & $132(100)$ & \\
\hline$>20$ & $16(64,0)$ & $9(36,0)$ & $25(100)$ & \\
\hline \multicolumn{4}{|c|}{ Tempo de TARV (anos) } & \multirow{5}{*}{0,016} \\
\hline$<5$ & $63(44,4)$ & $79(55,6)$ & $142(100)$ & \\
\hline $5-10$ & $40(50,6)$ & $39(49,4)$ & $79(100)$ & \\
\hline $11-20$ & $73(63,5)$ & $42(36,5)$ & $115(100)$ & \\
\hline$>20$ & $2(50)$ & $2(50)$ & $4(100)$ & \\
\hline Células T CD4 & $\mathrm{cel} / \mathrm{mm} 3)$ & & & \\
\hline$\geq 200$ & $167(52,5)$ & $151(47,5)$ & $318(100)$ & 0,819 \\
\hline$<200$ & $11(50)$ & $11(50)$ & $22(100)$ & \\
\hline Carga viral (có & $\mathrm{s} / \mathrm{ml})$ & & & \\
\hline$\leq 40$ & $149(54,2)$ & $126(45,8)$ & $275(100)$ & 0,165 \\
\hline$>40$ & $29(44,6)$ & $36(55,4)$ & $65(100)$ & \\
\hline
\end{tabular}


Ao avaliar a associação da presença do estresse com variáveis sociodemográficas e clínicas em PVHIV, observou-se que a idade $(p=0,018)$, o tempo de diagnóstico para o HIV ( $\mathrm{p}=0,025)$ e o tempo de uso de TARV ( $\mathrm{p}$ $=0,016$ ) foram variáveis que apresentaram associação significativa, como pode ser visto na tabela 3 acima.

Ao comparar homens e mulheres, identificou-se que houve maior predomínio de sinais e sintomas de estresse nas mulheres, independente da fase.

Observou-se, associação significativa dos sinais e sintomas com o sexo feminino na fase de alerta (Tabela 4), com destaque para mãos e/ou pés frios $(p=0,01)$, boca seca $(p=0,003)$, dor no estômago $(p=0,01)$, aumento da sudorese $(p=0,03)$ e outros.

$\mathrm{Na}$ fase de resistência e quase-exaustão (Tabela 5) também foi identificado associação significativa com o sexo feminino, com maior predomínio dos sinais e sintomas físicos, tais como problemas com a memória $(p=0,006)$, mal-estar generalizado $(0,005)$, formigamento de extremidades $(p=0,004)$, cansaço constante $(p=0,031)$ e presença de tontura na última semana $(\mathrm{p}<0,001)$.

Já na fase de exaustão, identificada pelos sinais e sintomas do último mês, houveram mais associações significativas entre o sexo feminino e os sintomas psicológicos (Tabela 6), dentre eles impossibilidade de trabalhar $(\mathrm{p}=0,03)$, vontade de fugir de tudo $(0,004)$ e pensar/ falar sobre o mesmo assunto $(\mathrm{p}=0,02)$

Tabela 4 - Sinais e sintomas físicos e psicológicos da fase de alerta segundo o sexo em PVHIV, n=340, Ribeirão Preto, SP, Brasil, 2016

\begin{tabular}{|c|c|c|c|c|c|}
\hline \multirow{2}{*}{\multicolumn{2}{|c|}{ Sinais e Sintomas }} & \multicolumn{2}{|c|}{ Sexo } & \multirow{2}{*}{$\begin{array}{l}\text { Total } \\
\mathrm{n}(\%)\end{array}$} & \multirow[b]{2}{*}{$\mathrm{p}$} \\
\hline & & $\begin{array}{l}\text { Masculino } \\
\mathrm{n}(\%)\end{array}$ & $\begin{array}{l}\text { Feminino } \\
\mathrm{n}(\%)\end{array}$ & & \\
\hline \multirow{12}{*}{ 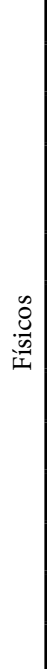 } & Mãos e/ou pés frios & $13(38,2)$ & $21(61,8)$ & $34(100)$ & 0,01 \\
\hline & Boca seca & $44(45,4)$ & $53(54,6)$ & $97(100)$ & 0,003 \\
\hline & Dor no estômago & $26(43,3)$ & $34(56,7)$ & $60(100)$ & 0,01 \\
\hline & $\begin{array}{l}\text { Aumento da sudo- } \\
\text { rese }\end{array}$ & $21(43,8)$ & $27(56,2)$ & $48(100)$ & 0,03 \\
\hline & Tensão muscular & $44(42,3)$ & $60(57,7)$ & $104(100)$ & $<0,001$ \\
\hline & $\begin{array}{l}\text { Aperto na man- } \\
\text { díbula }\end{array}$ & $22(73,3)$ & $08(26,7)$ & $30(100)$ & 0,07 \\
\hline & Diarreia passageira & $23(57,5)$ & $17(42,5)$ & $40(100)$ & 0,95 \\
\hline & Insônia & $66(58,9)$ & $46(41,1)$ & $112(100)$ & 0,79 \\
\hline & Taquicardia & $34(60,7)$ & $22(39,3)$ & $56(100)$ & 0,64 \\
\hline & Respiração ofegante & $33(60,0)$ & $22(40,0)$ & $55(100)$ & 0,73 \\
\hline & Hipertensão súbita & $07(33,3)$ & $14(66,7)$ & $21(100)$ & 0,01 \\
\hline & Mudança de apetite & $37(47,4)$ & $41(52,6)$ & $78(100)$ & 0,03 \\
\hline \multirow{3}{*}{ 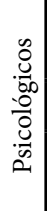 } & $\begin{array}{l}\text { Aumento súbito de } \\
\text { motivação }\end{array}$ & $25(43,1)$ & $33(56,9)$ & $58(100)$ & 0,01 \\
\hline & Entusiasmo súbito & $26(47,3)$ & $29(52,7)$ & $55(100)$ & 0,08 \\
\hline & $\begin{array}{l}\text { Vontade súbita de } \\
\text { outros projetos }\end{array}$ & $38(48,7)$ & $40(51,3)$ & $78(100)$ & 0,06 \\
\hline
\end{tabular}

Tabela 5 - Sinais e sintomas físicos e psicológicos da fase de resistência e quase-exaustão segundo o sexo em PVHIV, n=340,

Ribeirão Preto, SP, Brasil, 2016

\begin{tabular}{|c|c|c|c|c|c|}
\hline \multirow{2}{*}{ 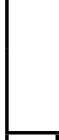 } & \multirow{2}{*}{ Sinais e Sintomas } & \multicolumn{2}{|c|}{ Sexo } & \multirow{2}{*}{$\begin{array}{l}\text { Total } \\
\mathrm{n}(\%)\end{array}$} & \multirow[b]{2}{*}{$\mathrm{p}$} \\
\hline & & $\begin{array}{l}\text { Masculino } \\
\mathrm{n}(\%)\end{array}$ & $\begin{array}{l}\text { Feminino } \\
\mathrm{n}(\%)\end{array}$ & & \\
\hline \multirow{10}{*}{ 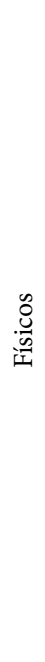 } & $\begin{array}{l}\text { Problemas com a } \\
\text { memória }\end{array}$ & $67(48,9)$ & $70(51,1)$ & $137(100)$ & 0,006 \\
\hline & Mal-estar generalizado & $37(44,6)$ & $46(55,4)$ & $83(100)$ & 0,005 \\
\hline & $\begin{array}{l}\text { Formigamento de } \\
\text { extremidades }\end{array}$ & $31(43,1)$ & $41(56,9)$ & $72(100)$ & 0,004 \\
\hline & $\begin{array}{l}\text { Sensação de desgaste } \\
\text { físico }\end{array}$ & $60(58,8)$ & $42(41,2)$ & $102(100)$ & 0,829 \\
\hline & Mudança de apetite & $41(50,0)$ & $41(50,0)$ & $82(100)$ & 0,094 \\
\hline & $\begin{array}{l}\text { Problema derma- } \\
\text { tológico }\end{array}$ & $21(60,0)$ & $14(40,0)$ & $35(100)$ & 0,794 \\
\hline & Hipertensão arterial & $08(50,0)$ & $08(50,0)$ & $16(100)$ & 0,510 \\
\hline & Cansaço constante & $49(49,0)$ & $51(51,0)$ & $100(100)$ & 0,031 \\
\hline & $\begin{array}{l}\text { Aparecimento de } \\
\text { úlcera }\end{array}$ & $25(58,1)$ & $18(41,9)$ & $43(100)$ & 0,978 \\
\hline & Tontura & $32(38,1)$ & $52(61,9)$ & $84(100)$ & $<0,001$ \\
\hline \multirow{5}{*}{ 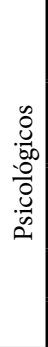 } & $\begin{array}{l}\text { Sensibilidade emotiva } \\
\text { excessiva }\end{array}$ & $70(53,6)$ & $63(47,4)$ & $133(100)$ & 0,112 \\
\hline & $\begin{array}{l}\text { Dúvidas quanto a si } \\
\text { próprio }\end{array}$ & $34(45,9)$ & $40(51,1)$ & $74(100)$ & 0,018 \\
\hline & $\begin{array}{l}\text { Pensamento sobre um } \\
\text { só assunto }\end{array}$ & $89(55,6)$ & $71(44,4)$ & $160(100)$ & 0,415 \\
\hline & Irritabilidade excessiva & $74(57,8)$ & $54(42,2)$ & $128(100)$ & 0,970 \\
\hline & Diminuição da libido & $55(48,2)$ & $59(51,8)$ & $114(100)$ & 0,010 \\
\hline
\end{tabular}

Tabela 6 - Sinais e sintomas físicos e psicológicos da fase de exaustão segundo o sexo em PVHIV, $\mathrm{n}=340$, Ribeirão Preto, SP, Brasil, 2016

\begin{tabular}{|c|c|c|c|c|c|}
\hline \multirow{2}{*}{\multicolumn{2}{|c|}{ Sinais e Sintomas }} & \multicolumn{2}{|c|}{ Sexo } & \multirow{2}{*}{$\begin{array}{l}\text { Total } \\
\mathrm{n}(\%)\end{array}$} & \multirow[b]{2}{*}{$\mathrm{p}$} \\
\hline & & $\begin{array}{l}\text { Masculino } \\
\mathrm{n}(\%)\end{array}$ & $\begin{array}{l}\text { Feminino } \\
\mathrm{n}(\%)\end{array}$ & & \\
\hline \multirow{8}{*}{ 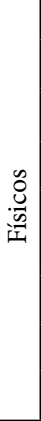 } & Diarreia frequente & $24(70,6)$ & $10(29,4)$ & $34(100)$ & 0,11 \\
\hline & Dificuldades sexuais & $14(42,4)$ & $19(57,6)$ & $33(100)$ & 0,05 \\
\hline & Náusea & $21(47,7)$ & $23(52,3)$ & $44(100)$ & 0,14 \\
\hline & Hipertensão confirmada & $11(34,4)$ & $21(65,6)$ & $32(100)$ & 0,005 \\
\hline & $\begin{array}{l}\text { Problemas dermatológicos } \\
\text { prolongados }\end{array}$ & $11(45,8)$ & $13(54,2)$ & $24(100)$ & 0,21 \\
\hline & Excesso de gases & $25(46,3)$ & $29(53,7)$ & $54(100)$ & 0,05 \\
\hline & Tontura frequente & $23(42,6)$ & $31(57,4)$ & $54(100)$ & 0,01 \\
\hline & Úlcera & $06(75,0)$ & $02(25,0)$ & $08(100)$ & 0,47 \\
\hline \multirow{8}{*}{ 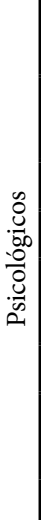 } & $\begin{array}{l}\text { Impossibilidade de tra- } \\
\text { balhar }\end{array}$ & $18(42,9)$ & $24(57,1)$ & $42(100)$ & 0,03 \\
\hline & $\begin{array}{l}\text { Sensação de incompetên- } \\
\text { cia em todas as áreas }\end{array}$ & $26(55,3)$ & $21(44,7)$ & $47(100)$ & 0,69 \\
\hline & Vontade de fugir de tudo & $63(48,1)$ & $68(51,9)$ & $131(100)$ & 0,004 \\
\hline & Apatia, depressão ou raiva & $58(55,8)$ & $46(44,2)$ & $104(100)$ & 0,59 \\
\hline & $\begin{array}{l}\text { Pensar/falar sobre o } \\
\text { mesmo assunto }\end{array}$ & $45(48,4)$ & $48(51,6)$ & $93(100)$ & 0,02 \\
\hline & Irritabilidade sem causa & $49(50,0)$ & $49(50,0)$ & $98(100)$ & 0,05 \\
\hline & $\begin{array}{l}\text { Angústia ou ansiedade } \\
\text { diária }\end{array}$ & $88(55,3)$ & $71(44,7)$ & $159(100)$ & 0,36 \\
\hline & Perda do senso de humor & $56(62,2)$ & $34(37,8)$ & $90(100)$ & 0,32 \\
\hline
\end{tabular}




\section{DISCUSSÃO}

O presente estudo evidenciou uma alta prevalência do estresse em PVHIV, destacando a associação entre o mesmo com a idade, o tempo de diagnóstico para o HIV e o tempo de tratamento com ARV. Além disso, demonstra, pela primeira vez, a associação de sintomatologias do estresse nessa população e ressalta como principal achado a associação entre sintomas físicos em mulheres que vivem com o HIV. A análise da sintomatologia do estresse, da maneira que foi abordada, ainda é incipiente na literatura, o que dificulta as comparações com outras fontes nessa temática.

Outro aspecto que se associa ao estresse é a idade. A maior prevalência do estresse foi encontrada em adultos jovens entre 30 e 39 . Tal fato pode ser justificado por se caracterizar como a fase inicial da progressão/descoberta da doença. $\mathrm{O}$ estresse e repercussões na qualidade de vida são encontrados entre pessoas infectadas pelo HIV, logo após o diagnóstico, com implicações negativas nos aspectos físicos e mentais (Huang et al., 2018). De maneira semelhante ao presente estudo, outra pesquisa identificou que um dos principais estressores estão associados ao diagnóstico do HIV, a revelação do mesmo e o início do tratamento. Tal condição de vida pode influenciar significativamente o desenvolvimento do humor depressivo (Jiang et al., 2019). A condução do tratamento terapêutico cotidiano, bem como seus efeitos colaterais são geradores de estresse (Cardoso, 2013). Deste modo, as implicações e o impacto na vida destas pessoas não devem ser negligenciados.

A inserção da TARV no contexto das PVHIV provocou aumento na expectativa e melhora na qualidade de vida; porém, viver com o HIV impôs a algumas pessoas limitações profissionais, sociais, afetivas, dificuldade de manter relacionamentos e empecilhos nas decisões reprodutivas. Assim, para quem vive com o vírus diversos aspectos da vida se tornaram uma situação de sofrimento, estresse e mal-estar (Ceccon, Meneghel \& Hirakata 2014).

Nesse contexto, sobre a perspectiva do HIV como condição crônica, é necessário transcender o cuidado pautado no tratamento farmacológico, para integrar todos os domínios do indivíduo em busca de uma assistência integral (Seidl e Faustino, 2014). Sob esta ótica, a condição psicossocial do indivíduo é imprescindível ao cuidado que visa atingir maior efetividade.

Segundo Seidl e Fautino (2014), ser soropositivo ao HIV é uma vivência subjetiva que constitui uma experiência singular frente à diversidade de reações psicológicas e emocionais que independem das possibilidades de tratamento e assim, para muitas PVHIV, o advento da TARV nem sempre modifica o curso da enfermidade. Apesar da maioria dos participantes $(58,0 \%)$ pertencer ao sexo masculino, ao comparar os sintomas de estresse entre homens e mulheres, o sexo feminino apareceu com maior frequência na fase de alerta $(60,8 \%)$ e na fase de quase-exaustão $(75,0 \%)$, ambos com predomínio dos sintomas físicos e na fase de exaustão $(54,8 \%)$ com predomínio de sintomas psicológicos.

Sintomas como boca seca, dor de estômago, dificuldades sexuais apresentaram associação significativa com o estresse. Sabe-se que situações de estresse ativam o eixo hipotalâmico-pituitário-adrenal (HPA) e o sistema nervoso autônomo para liberar hormônios do estresse sendo que sua exposição crônica resulta em respostas mal-adaptativas de vários órgãos, dando origem a adaptações fisiopatológicas indesejáveis inclusive de cunho cardiovascular (Brooks et al., 2018). Assim, a vivência subjetiva de viver com HIV transpõe a esfera psicológica para acometimentos fisiológicos que influenciam na qualidade de vida e que incita a discussão a respeito da demanda da interdisciplinaridade do cuidado. Quanto aos sintomas físicos e psicológicos, observou-se que nas fases de alerta e resistência, os físicos foram mais prevalentes entre as mulheres que vivem com HIV. Vale ressaltar, estudos que abordam a qualidade de vida nessa população, identificaram que as mulheres, também apresentam maiores escores de sintomatologia depressiva do que os homens, tanto no aspecto somático quanto no afetivo/cognitivo (Reis et al., 2017). Ademais, a epidemia de HIV/aids ainda é marcada pela desigualdade nas relações socioculturais estabelecidas entre os sexos. Sabe-se que há uma vulnerabilidade biológica e social das mulheres, a qual decorre da assimetria de poder entre os sexos, e que tem determinado a submissão feminina aos homens, a dificuldade em praticar sexo seguro, e ainda uma maior vulnerabilidade a violência, em comparação com aquelas que não vivem com o vírus (Jewkes, Dunkle, Nduna \& Shai, 2010; Kemppainen, MacKain, Alexander, Reid \& Jackson, 2017). Além das razões citadas, as manifestações dos sintomas psicológicos no estresse que se associam a mulher também pode estar relacionada com as responsabilidades as quais assumem de cuidados dos filhos ou do parceiro com HIV, o sustento da família, o preconceito e o estigma, gerando inclusive sofrimento emocional e pensamentos suicidas (Ceccon et al., 2014; Kemppainen et al., 2017). 
As consequências das desigualdades de poder entre os sexos, que vulnerabilizam as mulheres e as expõem a violências, acarretam o sofrimento emocional e pensamentos suicidas (Ceccon et al., 2014; Jewkes et al., 2010). Vale ressaltar que o estresse contribui na depressão da atividade do sistema imunológico, acelerando a progressão viral, facilitando o desenvolvimento da aids (Cardoso, 2013). Em recente meta-análise que avaliou a eficácia de diferentes tratamentos psicossociais para PVHIV com problemas de saúde mental, foi identificado que a realização de intervenções psicossociais teve um efeito positivo na depressão, ansiedade, qualidade de vida e bem-estar psicológico das pessoas que vivem com HIV (Luenen et al., 2018). Destaca-se ainda que não foram encontradas diferenças entre os vários tipos de intervenção, evidenciando que as mais diversas podem ser úteis para melhorar a saúde mental das PVHIV (Luenen et al., 2018). Neste mesmo sentido, Reis et al., (2017) afirma que a implementação de atividades de grupo direcionada à promoção da auto-estima e do compartilhamento de experiências pode ampliar e fortalecer fontes de apoio social, trabalhando como um fator de proteção contra vulnerabilidades em saúde mental. O suporte social por sua vez é um importante fator de integração social entre estes indivíduos, influenciando o conviver com o HIV da melhor maneira diariamente e, por isso o mesmo, é considerado como ponto chave para a prevenção e recuperação de doenças, promoção da saúde e adesão terapêutica (Galvão et al., 2015). No processo saúde- doença, o suporte social é o moderador do estresse e auxilia no enfrentamento participativo entre indivíduos com HIV, profissional da saúde e rede social de apoio, nas diversas dificuldades encaradas durante a vida (Calvetti et al., 2016).

Destaca-se ainda que as demandas apontadas pelas pessoas que vivem com a infecção pelo HIV, requerem autocuidado contínuo, aprendizado de diversos aspectos clínicos, e mudanças de atitudes e de comportamentos que favoreçam o enfrentamento dessas demandas advindas do viver na perspectiva de condição crônica.

\section{CONCLUSÕES}

O estresse entre as PVHIV apresentou uma alta prevalência, associação entre idade, tempo de diagnóstico e de TARV, e predomínio entre sinais e sintomas nas mulheres. Tais dados alertam para a necessidade de ações voltadas para prevenção do estresse, o qual pode gerar prejuízos significativos no cotidiano, na adesão e evolução do tratamento e na qualidade de vida.
O acompanhamento multiprofissional é fundamental na prevenção e tratamento, e a avaliação das manifestações físicas e psicológicas pode ser realizada durante o atendimento hospitalar, domiciliar ou ambulatorial.

\section{IMPLICAÇÕES PARA A PRÁTICA CLÍNICA}

A equipe multiprofissional enfrenta novos desafios diante da complexidade das demandas advindas da cronicidade do viver com HIV, que inclui não só a reconstrução de projetos de vida, mas a crescente busca por melhorar a qualidade de vida desses indivíduos em todos os seus aspectos. Assim, espera-se que o estudo subsidie a construção de espaços de diálogo e que esses espaços permitam a criação de vínculos e promova a escuta qualificada para as necessidades dessa população, sobretudo para as mulheres, cujos papéis relacionados ao trabalho e à família têm diferentes significados.

\section{REFERÊNCIAS BIBLIOGRÁFICAS}

Brasil. Ministério da Saúde. (2018). Boletim Epidemiológico HIV AIDS 2018. Brasília: Ministério da Saúde.

Brooks, S., Brnayan, K.W., DeVallance, E., Skinner, R., Lemaster, K., Sheets, J.W., Pitzer, C.R., Asano, S., Bryner R.W., Olfert, I.M, Frisbee JC, \& Chantler PD. Psychological stress induced cerebrovascular dysfunction: The role of metabolic syndrome and exercise. Experimental Physiology, 103(5), 761-776. Doi: 10.1113/EP086892

Calvetti, P.U., Giovelli, G.R.M., Gauer, G.J.C., e Moraes, J.F.D. (2016) Níveis de Ansiedade, Estresse Percebido e Suporte Social em Pessoas que Vivem com HIV/Aids. Psicologia: Teoria e Pesquisa, 32(4), e324317. Doi: $10.1590 / 0102.3772 \mathrm{e} 324317$

Cardoso, G.S.S. (2013). Manejo de estresse para pacientes com HIV/AIDS por meio da TCC. Revista Brasileira de Terapias Cognitivas, 9(1), 26-33. Doi: 10.5935/18085687.20130005

Ceccon, R.F., Meneghel, S.N., e Hirakata, V.N. (2014). Mulheres com HIV: violência de gênero e ideação suicida. Revista de Saúde Pública, 48(5), 758-765.

Collado-Hidalgo, A., Sung, C., \& Cole, S. (2006) Adrenergic inhibition of innate antiviral response: PKA blockade of Type I interferon gene transcription mediates catecholamine support for HIV-1 replication. Brain, Behavior, and Immunity, 20(6), 552-563. 
Foresto, J.S., Melo, E.S., Costa, C.R.B., Antonini, M., Gir, M., e Rei, R.K. (2017). Adesão à terapêutica antirretroviral de pessoas vivendo com HIV/aids em um município do interior paulista. Revista Gaúcha de Enfermagem, 38(1), e63158. Doi: 10.1590/19831447.2017.01.63158

Galvão, M.T.G., Soares, L.L., Pedrosa. S.C., Fiuza, M.L.T., e Lemos. L.A. (2015) Qualidade de vida e adesão à medicação antirretroviral em pessoas com HIV. Acta Paulista de Enfermagem, 28(1), 48-53. Doi: 10.1590/1982-0194201500009

Huang, Y., Dan, L., Chen, X., Zhang, D., Wang, M., Qiu, Y., Liu, Y., Peng, B., Niu, L., Xiao, S. (2019) Changes and determinants of health-related quality of life among people newly diagnosed with HIV in China: a 1-year follow-up study. Quality of Life Research, 28, 35-46. Doi: $10.1007 / \mathrm{s} 11136-018-1998-\mathrm{x}$

Jewkes, R.K., Dunkle, K., Nduna, M., \& Shai, N. (2010) Intimate partner violence, relationship power inequity, and incidence of HIV infection in young women in South Africa: a cohort study. The Lancet, 376(9734), 41-48. Doi: 10.1016/S0140-6736(10)60548-X

Jiang, H., Tan, Z., Cheng, W., Xu, M., Lin, P., Huang, X, Huang, R, Liu, J., Yang, F., Li, Y., Gao, Y., Yang, Y. (2019) Negative life events and major depressive disorder among HIV-positive individuals in Guangdong, China. Medicine, 98(37), e17174. Doi: 10.1097/ MD.0000000000017174.

Joint United Nations Programme on HIV/AIDS. (2016). On the fast track to ending the AIDS epidemic. [Adobe Digital Edition version].

Kemppainen, J.K., MacKain, S., Alexander, M., Reid, P., \& Jackson, M.P. (2017) Posttraumatic Stress Disorder and Stressful Life Events Among Rural Women With HIV Disease. Journal of The Associaton of Nurses in AIDS Care, 28(2), 216-25. Doi: 10.1016/j. jana.2016.06.001

Lipp, M.E.N. (2000). Manual do Inventário de Sintomas de Stress para Adultos de Lipp (ISSL). São Paulo: Casa do Psicólogo.

Lipp, MEN., e Malagris, L.N. (1989). Manejo do estresse. In Rangé, B. (Eds.), Psicoterapia comportamental e cognitiva (pp.279-282). Campinas, SP: Psy.
Lipp MEN., Guevara, A.J.H. Validação empírica do Inventário de Sintomas de Stress (ISS). Estudos de Psicologia (Campinas), 1994; 11(3):43-9.

Reis, R.K., Castrighini, C.C., Melo, E.S., Jesus, G.J., Queiroz, A.A.F.L., e Gir, E. (2017) Avaliação dos sintomas depressivos somáticos e afetivo-cognitivos de pessoas vivendo com HIV/AIDS. Acta Paulista de Enfermagem, 2017; 30(1): 60-65.

Seidl, E.M.F. e Faustino, Q.M. (2014). Pessoas vivendo com HIV/Aids: Possibilidades de atuação da Psicologia. In Seidl, E.M.F. e Miyazaki, M.C.O.S. (Eds.), Psicologia da Saúde: Pesquisa e atuação profissional no contexto de enfermidades crônicas (pp. 21-31). Curitiba: Juruá. Selye, H. (1956). The Stress of life. New York: Mc Graw.

Stults-Kolehmainen, M.A., Bartholomew, J.B., \& Sinha, R. (2014). Chronic psychological stress impairs recovery of muscular function and somatic sensations over a 96-hour period. Journal of Strength and Conditioning Research, 28(7), 2007-2017. Doi: 10.1519/ JSC.0000000000000335.

Ursoiu, F., Moleriu, L., Lungeanu, D., \& Puschită, M. (2018) The association between HIV clinical disease severity and psychiatric disorders as seen in Western Romania. AIDS Care,30(11), 1368-1371. Doi: 10.1080/09540121.2018.1455959.

Van Luenen, S., Garnefski, N., Spinhoven, P., Spaan, P., Dusseldorp, E., \& Kraaij, V. (2018). The Benefits of Psychosocial Interventions for Mental Health in People Living with HIV: A Systematic Review and Meta-analysis. AIDS and Behavior, 22, 9-42.

Verhey, R., Gibson, L., Brakarsh, J., Chibanda, D., \& Seedat, S. (2018). Prevalence and correlates of probable post-traumatic stress disorder and common mental disorders in a population with a high prevalence of HIV in Zimbabwe. European Journal of Psychotraumatology, 9,1536286. Doi: 10.1080/20008198.2018.1536286.

\section{Fontes de Financiamento}

Agradecemos ao Ministério da Ciência, Tecnologia e Inovação - Conselho Nacional de Desenvolvimento Científico e Tecnológico (CNPq) (n 455912/2014-9) - Chamada Universal - MCTI/CNPq no 14/2014 e a Coordenação de Aperfeiçoamento de Pessoal de Nível Superior - Brasil (CAPES) - Código de Financiamento 001, pelo apoio na realização deste estudo. 